\title{
The Correlations between Planetary Nebula Morphology and Central Star Evolution. Analysis of the Northern Galactic Sample.
}

\author{
Letizia Stanghellini ${ }^{1,2}$ \\ Eva Villaver ${ }^{1}$ \\ Arturo Manchado 3,4 \\ Martin A. Guerrero ${ }^{5}$
}

\begin{abstract}
Northern Galactic Planetary nebulae (PNs) are studied to disclose possible correlations between the morphology of the nebulae and the evolution of the central stars (CSs). To this end, we have built the best database available to date, accounting for homogeneity and completeness. We use updated statistical distances, an updated morphological classification scheme, and we calculate Zanstra temperatures for a large sample of PNs. With our study we confirm that round, elliptical, and bipolar PNs have different spatial distributions within the Galaxy, with average absolute distances to the Galactic plane $0.73,0.38$, and $0.21 \mathrm{kpc}$ respectively. We also find evidence that the distributions of the central star's masses are different across these morphological groups, although we do not find that CSs hosted by bipolar PNs are hotter, on average, than CSs within round and elliptical PNs. Our results are in broad agreement with the previous analyses, indicating that round, elliptical, and bipolar PNs evolve from progenitors in different mass ranges, and may belong to different stellar populations, as also indicated by the helium and nitrogen abundances of PNs of different morphology.
\end{abstract}

\footnotetext{
${ }^{1}$ Space Telescope Science Institute, 3700 San Martin Drive, Baltimore MD 21218, USA; lstanghe@stsci.edu; villaver@stsci.edu

${ }^{2}$ Affiliated to the Astrophysics Division, Space Science Department of ESA; on leave, INAF- Osservatorio Astronomico di Bologna

${ }^{3}$ Instituto de Astrofisica de Canarias, via Lactea s/n, La Laguna, E-38200 Santa Cruz de Tenerife, Spain; amt@iac.es

${ }^{4}$ Consejo Superior de Investigaciones Cientificas, Spain

${ }^{5}$ Department of Astronomy, University of Illinois at Urbana-Champaign, 1002 W. Green St., Urbana, IL 61801; mar@uiuc.edu
} 
Subject headings: Planetary Nebulae: Morphology, Evolution. Central stars: Progenitors, Evolution

\section{Introduction}

Stars with masses in the $1-8 \mathrm{M}_{\odot}$ range are very likely to go through the evolutionary phase of $\mathrm{PN}$ ejection at the end of their lives. The ejection of most of the gaseous envelope in Asymptotic Giant Branch (AGB) stars occurs at the tip of the Thermal Pulse phase, in the form of a massive, low velocity wind (Iben \& Renzini 1983). Subsequently, the remnant CS ionizes the gaseous ejecta, while the fast, low mass-loss rate CS wind shapes the PN. The observed PN morphology thus depends on a complicate combination of phenomena, some occurring within the nebular gas, which evolves in dynamic time scale, others deriving from the evolution of the stellar progenitors and of the CSs. Morphology may also depend on the physical status of the interstellar environment of the PN progenitor.

The relations between PN morphology and CS evolution may be used to explore the gas versus star interplay. Pioneering this field, Peimbert (1978) found a link between PN morphology and stellar evolution through the chemical elements that are produced by PN progenitors, and are drawn to the stellar surface via dredge-up episodes. Peimbert's study showed that most of the helium- and nitrogen-rich, carbon-poor PNs where of asymmetric (bipolar etc.) shapes. This result is broadly consistent with the predictions of stellar evolution if the progenitors of asymmetric PNs have on average larger masses than the progenitors of round (and elliptical) PNs, without any assumption or relation to stellar multiplicity of the stellar progenitors.

About a decade ago, several authors have tried to tie up the correlations between nebular morphology and stellar properties (Stanghellini, Corradi, \& Schwarz 1993; Corradi \& Schwarz 1995), inspired by the new bounty of narrowband images available for Galactic PNs (Schwarz, Corradi, \& Melnick 1992; Balick 1987). Stanghellini, Corradi, \& Schwarz (1993), based on a large, yet non homogeneously selected, sample of Southern PNs from the ESO catalog (Schwarz, Corradi, \& Melnick 1992), found that the CSs of symmetric and asymmetric PNs have different mass distribution, and that the CSs of bipolar PNs are spread over a large mass range. Other papers have been written in the recent years (Amnuel 1995; Gorny, Stasinska, \& Tylenda 1997) on this subject, none of which have challenged the earlier results.

Strength to the association of bipolar PNs with high mass progenitors comes from

the Galactic distribution of morphological types. Among others, Schwarz, Corradi, \& Stanghellini (1993), Stanghellini (1995), and Manchado et al. (2000) found that bipolar 
PNs lie closer to the Galactic plane than round and elliptical PNs, making the high-mass origin plausible.

The motivation of the present work is to test the correlations between PN morphology and CS evolution with a larger and homogeneous PN database. In 1996 the IAC Catalog of PNs (Manchado et al. 1996), and with it a complete and homogeneous PN image data set, became available.

The aim of this paper is to discuss the relations between morphological type and the physical parameters that are linked to stellar populations and evolution. We will examine principally the variations of temperature, luminosity, and mass of the CSs. We will also show the variation across morphological types of elemental abundances and Galactic distribution. The statistics of the nebular characteristics variations across morphological types will be published elsewhere (Manchado et al. 2002).

In $\S 2$ we discuss the sample selection and the morphological classification. In $\S 3$ we describe the determination of the stellar temperature and luminosity. In $\S 4$ we present the results of our analysis. A discussion of the significance of our findings for the understanding of the evolution of PNs and their CSs is presented in $\S 5$.

\section{Morphological classification}

The database that we have used in this paper includes the IAC morphological catalog of Northern Galactic PNs (Manchado et al. 1996), supplemented by a selection of PNs from Balick (1987) and Schwarz, Corradi, \& Melnick (1992). Therefore, our sample includes all PNs in Acker et al. (1992) that are larger than 4 arcsec in maximum size, and that are within the observing limits of the IAC and La Palma Observatory telescopes (Manchado et al. 2000).

We classify the PN morphology primary on the basis of the $\mathrm{H} \alpha$ images, and secondary on the [O III] 5007 and the [N II] 6584 images, when available. A thorough discussion on the

extended morphological classification will be available in Manchado et al. (2002). Here, we briefly describe the morphological classes used to analyze the results on the CSs correlations.

We use the following, simplified morphological scheme:

- Round (R) PNs.

- Elliptical (E) PNs. We distinguish between R and E PNs if an axial difference of at least $5 \%$ is detected. 
- Bipolar (B) PNs. As commonly defined, bipolarity implies at least one pair of lobes and a pinched waist. Quadrupolar PNs belong to this category.

Together with the three main morphological classes above, we also examine the group properties of Bipolar Core (BC) PNs. This morphological class identifies those PNs whose external contour does not show obvious bipolar lobes, but whose inner structure is clearly suggestive of a projected ring. A ring-like structure was detected above the $20 \%$ flux level for our BC PNs to be classified as such. This class was firstly used in Stanghellini et al. (1999) to analyze bipolarity in Magellanic Cloud PN images acquired before the first HST servicing mission that restored the telescope optics. Note that Gorny, Stasinska, \& Tylenda (1997) use a similar definition for embedded bipolars (BE).

A few comments on this simplified classification scheme are in order. First note that Stanghellini, Corradi, \& Schwarz (1993) do not distinguish between R and E PNs. We now know that it is possible to separate these classes, and that they have different properties. Note that our classification of round PN is an objective one, based on the measured axial ratio on the $2 \mathrm{D}$ images, without making assuptions on the 3D structures (note that Soker (2002) uses a different classsification approach, and subjectively defines elliptical those PNs that have structures such as knots or filaments, even if they have round contours).

Second, in this paper we subsume the quadrupolar PNs into the bipolar class. Quadrupolar (Q) PNs show two pairs of lobes (Manchado, Stanghellini, \& Guerrero 1996) and their formation mechanism has to be similar to that of bipolar PNs. Our database contains but a few Q PNs, and studying them as a class apart will dilute their statistical significance anyway.

Third, we do not classify pointsymmetric (P) PNs as a separate morphological type. As pointed out by Manchado et al. (2000), pointsymmetric structures are present in a variety of main body morphologies. For this reason, we classified the pointsymmetric PNs as round, bipolar, or elliptical depending on the structure of the main body of the PN. Pointsymmetry is detected in a very small fraction of the PNs in our sample, and if they were selected in a separate class the results from our analysis would not change statistically. Finally, it is worth noting that the bipolar core PNs would have been classified as R or E in the usual or the new morphological schemes (Manchado et al. 2002). We want to isolate their properties in this paper, and relate them to those of B PNs. By doing so, we moderately decrease the $\mathrm{R}$ and $\mathrm{E} P \mathrm{P}$ data sets. 


\section{Temperature and luminosity derivation}

The stellar properties of the selected PNs were determined via the Zanstra analysis (Kaler 1983). The input parameters to the Zanstra analysis were selected from the literature, with the endeavor of using the best possible set of input data, chosen as homogeneously as possible. In Table 1 we list our reference choices for the input parameters.

The stellar magnitudes used for the analysis were taken, for the most part, from the paper by Tylenda et al (1991).

The $\mathrm{H} \beta$ and He II ( $\lambda 4686)$ line intensities were drawn, when available, from Cahn, Kaler, \& Stanghellini (1992), otherwise from Tylenda et al (1991). Additional fluxes and extinction constant references are listed in Table 1. Extinction constants are from Cahn, Kaler, \& Stanghellini (1992) or, alternatively, from Stasinska et al. (1992). If neither of these two references had relevant data, we used the additional references listed in table 1.

Nebular radii were measured by us on the original frames. For elliptical PNs, we compute equivalent radii as $R_{\mathrm{E}}=[(a \times b) / 4]^{1 / 2}$, where $a$ and $b$ are the elliptical axes. For bipolar PNs, we make the approximation that the lobes are two ellipses, and we define $R_{\mathrm{E}}=[(a \times b) / 4]^{1 / 2}$, where $a$ is the extension of the waist, and $b$ the extension of the bipolar lobes.

All distances used in this paper are statistical distances. We have calculated all the statistical distances based on the newly measured radii, and the Cahn, Kaler, \& Stanghellini (1992) distance scale calibration. The $6 \mathrm{~cm}$ fluxes used in this calculation were taken from Cahn, Kaler, \& Stanghellini (1992) where available, otherwise from the other references in Table 1. To assure that the calibration of the distance scale is independent on the morphological type of the calibrators used, we have morphologically classified the calibrators in Cahn, Kaler, \& Stanghellini (1992), and verified that all morphological types have similar weight in the calibration. We also verified that the calibration based on just bipolar and bipolar core PNs is almost identical to the calibration based on round PNs.

All input data are used in our Zanstra code with their own original error bars, as given from the original papers listed above and in Table 1. The Zanstra analysis then produces the propagated error bars. The statistical distances do not carry error bars, as it is impossible to quantify the magnitude of the errors in the assumptions made to scale the ionized masses with the nebular distances (see the discussion by Cahn, Kaler, \& Stanghellini (1992)). 


\section{Results}

In the following sections we illustrate the results of our investigation. Among the 255 Northern Galactic PNs with morphological classification, Zanstra analysis was performed in all the cases where the input parameters were available in the literature, and precisely for 54 round, 96 elliptical, 36 bipolar, and 19 bipolar core PNs.

In Table 2 we give the results of the Zanstra analysis. Column (1) gives the PN name; column (2) gives the morphological type, following the simplified scheme described in $\S 2$. Unless otherwise noted within the Table the images for morphological analysis were from Manchado et al. (1996). Columns (3) through (6) give the Zanstra temperature (and error) and luminosity (and error), in logarithmic format. We always give the He II Zanstra temperature if available. Otherwise, we give the $\mathrm{H}$ I Zanstra temperature. In the cases in which the stellar magnitude was not available, we give the cross-over temperature instead (Kaler \& Jacoby 1989). The reader should not use cross-over temperatures in Table 2 at face value, since they are calculated assuming that the He II and H I temperatures are equal. Cross-over temperatures are upper limits for most nebulae except for optically thick PNs, and the magnitudes estimated via cross-over analysis are also upper limits.

Distances, dimensions, and detailed morphology will be discussed in a future paper.

In Table 3 and 4 we give the averaged physical diagnostics across morphological types that we use in the following sections. Table 3 contains the Galactic distribution and Zanstra temperature diagnostics (with the $\sigma$ values in square braketts), while Table 4 gives the elemental abundance diagnostics.

\subsection{Spatial distribution}

The apparent spatial distribution of Galactic PNs with different morphology is shown in Figure 1, where the different symbols indicate morphological types (open circles are round, asterisks are elliptical, filled squares are bipolar, and open squares are bipolar core PNs). It appears from this plot that the bipolar and bipolar core PNs are found at lower Galactic latitudes, and their distribution do not extend, for the most part, away from the disk. Differently, elliptical and round PNs are spread all over the l-b plane. From column (2) in Table 3 we can see that the round PNs have a latitude range much larger than that of bipolar PNs.

We have run the Kolmogorov-Smirnov (K-S) statistics (Presse et al. 1986) on the Galactic latitude distribution to investigate the quantitative differences of the different morphology 
PNs. The K-S statistical test give the maximum absolute value of the difference between two given distributions, $\mathrm{D}_{\max }$. The significance level for the null hypothesis that the data sets are drawn from the same distribution $(\mathrm{P})$ is also given by the K-S statistics: lower values of $\mathrm{P}$ indicate two different distributions. Typically, $\mathrm{P} \leq 5 \times 10^{-2}$ indicates that there is a real possibility that the two distributions are different, while $\mathrm{P}=1$ indicates identical distributions. Larger data sets give better results, but data sets as large as $\mathrm{N}=20$ already give a good indication on the statistics.

By considering the Galactic latitude distributions of $\mathrm{R}$ and $\mathrm{B}$ PNs, we found that $\mathrm{D}_{\max }=1.8$ and $\mathrm{P}=0.003$. The latitude distributions of round and elliptical PNs are not so sharply dissimilar $(\mathrm{P}=0.19)$. To enhance the statistical significance of the results we grouped $\mathrm{B}$ and $\mathrm{BC} \mathrm{PNs}$ in one class $(\mathrm{B}+\mathrm{BC})$. The K-S probability for $\mathrm{R}$ and $\mathrm{B}+\mathrm{BC} \mathrm{PNs}$ to have similar $b$ distributions is 0.086 , while this probabilities is 0.53 when we use the $\mathrm{E}$ and $\mathrm{B}+\mathrm{BC}$ samples. Our conclusion is that there is a sharp difference between the nature of $\mathrm{R}$ and $\mathrm{B}+\mathrm{BC}$ PNs. Much more detail on these distribution will be presented in a future paper.

In Figure 2 we show the PNs distribution of the absolute distance to the Galactic plane, $|z|$, for each morphological type. This parameter is calculated with the statistical distance scale, and the sample sizes are different than those of Figure 1, since some PNs did not have the necessary parameters in the literature for the derivation of their statistical distance. We see in Figure 2 that the distribution of R PNs is the broadest, followed by the E, the BC, and the B PNs.

We have calculated the average scale distance from the Galactic plane for the different morphological types (column [3] in Table 3). Round PNs have the largest average $|z|, \mathrm{E}$ PNs follow, then BC PNs, and finally B PNs, closest to the Galactic plane. The average scale height of all PNs in the sample is $0.44 \mathrm{kpc}$. Our calculations basically confirm the early results by Schwarz, Corradi, \& Stanghellini (1993). It is worth noting that the average distance per morphological class are comparable to one another.

Our results on the Galactic distribution of morphological types show that morphology could be used as indicator of stellar population. For example, the scale height for a young disk population is similar to that of bipolar and bipolar core PNs (Scalo 1998). The caveat of this conclusion is that there may be a selection effect that limits the observations of low surface brightness PNs toward the Galactic plane.

In order to asses the importance of the selection effects, we checked the $\mathrm{H} \beta$ surface brightness distributions of the PNs, calculated as $\mathrm{F}_{\beta} / \pi R^{2}$, where the $\mathrm{H} \beta$ flux is corrected for extinction and the radii are the equivalent radii calculated as described in $\S 3$. We found that surface brightness of $\mathrm{R}$ and $\mathrm{E}$ PNs are in the $-17<\log S B_{\beta}<-11.5$ range, while 
$\mathrm{B}$ and BC PN's surface brightness range within $-15.5<\log S B_{\beta}<-12.5^{6}$. The lack of high surface brightness B and BC PNs may simply indicate that they evolve faster than $\mathrm{E}$ and R PNs, similarly to what observed in the Magellanic Clouds (Stanghellini et al. 1999; Shaw et al. 2001). This is another hint that B and BC PNs may originate from higher mass progenitors.

On the other hand, the lack of very low surface brightness B and BC PNs may indicate that there may be a selection effect against low surface brightness B and BC PNs in the Galaxy, possibly due to the interstellar extinction which is higher for these PNs, given their location within the Galactic plane. The average $\mathrm{H} \beta$ extinction for each morphological type is given in column (4) or Table 3. Since on average extinction does anti-correlates with the distance from the Galactic plane, it roughly indicates that interstellar extinction is predominant, and the internal extinction is only marginally important in the overall $\mathrm{c}_{\beta}$ values.

\subsection{Central stars on the $\log \mathrm{L}-\log \mathrm{T}$ plane, and the inferred mass distribution}

Zanstra temperatures of the CSs were calculated as described by Stanghellini, Corradi, \& Schwarz (1993). The He II temperatures are the most reliable, as most PNs are optically thick to the He II ionizing photons. H I temperatures are generally reliable for the optically thick PNs. When using H I Zanstra temperatures we should keep in mind that it is difficult to define which $\mathrm{PN}$ is optically thick and which is thin, especially in the case of bipolar PNs. Thus the only reliable results are the few temperatures calculated from the He II line intensities.

In order to disclose all possible relations between PNs and their CSs, we have taken a conservative approach. We first consider the most reliable Zanstra temperature set, using only He II Zanstra temperatures with minimal errors $\left(\Delta \log T_{\text {eff }}<0.1\right)$. The averages of column (5) in Table 3 refer to this set. We do not find marked differences among the average temperatures of stars hosted by different morphological types.

Corradi \& Schwarz (1995) found an average effective temperature of 142,000 K for stars hosted by bipolar PNs, and 99,000 for those within the elliptical (including round) PNs. We do not disclose such a difference in the average temperatures. In Figure 3 we show the location on the $\log \mathrm{T}-\log \mathrm{L}$ plane of the PN CSs, where $\Delta \log T_{\text {eff }}<0.1$ and $\Delta \log L / L_{\odot}<0.3$

\footnotetext{
${ }^{6}$ This results is obtained with the PNs of Table 2, but we eliminated NGC 7017 from this calculation
} 
(note that the error bars do not account for uncertainties in the statistical distances).

The HR diagram distribution of Figure 3 shows that stars hosted by round nebulae are spread on the $\log \mathrm{T}-\log \mathrm{L}$ plane differently than stars hosted by bipolar and bipolar core PNs. Central stars of elliptical PNs cover more or less the whole plane, up to the $0.8 \mathrm{M}_{\odot}$ evolutionary track. We derive the distribution of the CS's masses for the different morphologies from Figure 3, and we show it in Figure 4. Round nebulae appear to have a peak CS mass around 0.55, then the distribution declines for larger masses. Central stars of elliptical PNs have a flatter mass distribution. Bipolar and bipolar core PNs seem to have a flat mass distribution (better compare the round and elliptical distribution with the sum of bipolar and bipolar core, since these two later classes are so sparsely populated).

Figure 5 is similar to Figure 3, with the difference that we plot the HR diagram location of CS PNs whose temperatures have errors smaller than 0.4 dex, and where we include the $\mathrm{H}$ I Zanstra temperatures. Figure 6 gives the histograms of the mass distributions relative to the sample of Figure 5. Here, the low-mass section of the histogram for elliptical PNs is more prominent than in Figure 4, but the overall distributions have the same qualitative form. These results are indicative of different CS masses for different PN shapes. The statistical K$\mathrm{S}$ analysis applied to this set of temperatures (and the relative luminosities) does not disclose enormous differences between the effective temperature and luminosity distributions.

We have analyzed the ratios of He II to H I Zanstra temperatures across morphological types, to have an indication of their optical thickness. Our aim was to probe the preliminary results of Figures 1 and 3 in Stanghellini, Corradi, \& Schwarz (1993), by using the better and more homogeneous sample of Northern Galactic PNs. Our results (column [6] of Table 3) shows that, although there are differences in the average Zanstra ratio for the various morphologies, such differences are milder that previously found (Stanghellini, Corradi, \& Schwarz 1993). We confirm that, on average, bipolar PNs have lower Zanstra ratio than symmetric PNs, but we can not draw the same conclusion for bipolar core PNs based on our small sample (see also the higher sigma value).

\subsection{Hints from elemental abundances}

During the evolution of intermediate-mass stars, the chemical enrichment of the outer region of the stars occurs in a series of dredge-up and nuclear burning events (Iben \& Renzini 1983), that enrich the future PN in different ways, depending on the stellar mass. By measuring the CNO elemental abundances we can probe stellar evolution. Carbon depletion and nitrogen enrichment depend on the progenitor mass, forming a direct connection between 
observed progenitor mass (i.e. Population) and chemical content.

The argon and neon abundances in PNs are probes of the original chemical mix at the time of the formation of the progenitors. In fact, these elements are unaffected during the evolution of the stars in this mass range.

A large study of the PN abundances and their correlations to shell morphology is in preparation (Manchado et al. 2002), based on abundances of Galactic PNs newly calculated with a selection of the available spectral data in the literature. In Table 4 we show the averages of the derived abundances (all abundances are with respect to hydrogen, by number), calculated for each morphological type. Note that we use only elemental abundances with low errors (see Manchado et al. (2002)). With each entry, in parenthesis, we report the statistical sample used for that particular diagnostic.

Among the significant results, we find that helium abundances are, on average, higher in bipolar PNs than in round and elliptical PNs. The average helium abundance trace nicely the increasing asymmetry, from round to bipolar PNs. If we consider $\mathrm{R}+\mathrm{E} \mathrm{PNs}$ and $\mathrm{B}+\mathrm{BC}$ PNs, the average helium abundance of the two groups differ, quite significantly, at the 25 percent level.

The oxygen abundance averages do not change as much from one group to the next, with the exception that the average oxygen abundance for bipolar PNs is slightly higher than for the other groups.

Bipolar PNs have much higher average nitrogen abundance than the other morphological types, confirming the results by Peimbert (1978) and others. Bipolar core PNs are mildly nitrogen enriched, based on a rather small PN sample. If we compare the $\mathrm{R}+\mathrm{E}$ and the $\mathrm{B}+\mathrm{BC}$ groups, we obtain that the average nitrogen abundances in the two groups differ at the 60 percent level. This is a clear signature that bipolar PNs have massive progenitors.

Other diagnostics do not show remarkable differences, or are statistically insignificant. We do not see the trend found in LMC, where B PNs have higher neon and argon abundances (Stanghellini, et al. 2000).

While the abundances used here are probably the best collection available to date, it is very difficult to draw sound conclusions on the basis of sparse spectroscopic data with slits collecting variable areas of PNs. This is particularly debilitating for multiple shell or very bipolar PNs, as their abundance gradients can be substantial. It is worth remarking that, in addition to the above difficulties, the abundances are also contaminated by the ISM material, ingested by the PN during their evolution (Villaver, Garcia-Segura, \& Manchado 2002). 


\subsection{Evolutionary paths}

As seen in the two preceding sections, there is an indication of mass segregation among the CSs hosted by symmetric and asymmetric PNs. This has bearings on the possible evolutionary paths that lead to the different morphologies. The formation of asymmetric PNs has been ascribed to binary evolution, either through common envelope (CE) or via wide binary or planetary companions (Soker 1997), as well as to the presence of stellar rotation and magnetic fields (Garcia-Segura, Langer, \& Rozyczka 1999). Soker (1997) selected a large sample of PNs, and, based solely on their morphology, determines the likeliness that these morphologies originate (1) from the evolution of a single star, (2) from binary evolution, where the member of the binary system go through the Common Envelope (CE) phase, (3) from binary evolution of a pair that avoids the CE phase, or (4) from the evolution of a star and a planet companion. We have checked Soker's determinations of the stellar origins of PNs against our morphological sample. We found, based on our classification scheme and on his evolutionary scheme, that (1) no B or BC PNs would originate from single stars; (2) no round PNs progenitors would have gone through the CE phase; and (3) no R nor E PNs would originate through binary stars, without going through the CE phase. If (1) was true, then why the CS masses of B and BC PNs are distributed differently than those of $\mathrm{R}$ and $\mathrm{E}$ PNs? Why B and BC PNs would be found at lower scale heights on the Galactic plane, and would they have higher helium and nitrogen content? Clearly, a theoretical scenario that explains all the observations is not available at this time. In particular, while the binary star scenario can be streched to explain the Galactic distribution of bipolar PNs (Soker 1998), much more stellar evolution modeling is needed to compare the chemical yields in the cases of single and binary star evolution.

\section{Summary and discussion}

The analysis of Northern Galactic PNs have produced interesting results. First, there is a general indication that bipolar and bipolar core PNs belong to the same physical class, even if they may be at different evolutionary stages. This results from the apparent and

physical spatial distribution of $\mathrm{B}$ and $\mathrm{BC}$ PNs, from the excinction analysis, and from the CSs distributions. The BC sample is barely large enough for accurate statistical analysis, thus this result is still to be confirmed in the future. It agrees, though, with the analysis of Magellanic Cloud PNs, as discussed by Stanghellini et al. (1999); Stanghellini, et al. (2000); Shaw et al. (2001).

Second, we have analyzed the CSs via Zanstra analysis. The results are in moderate agreement with those of Stanghellini, Corradi, \& Schwarz (1993), after correcting for the 
different morphological classification. Although the mass histograms of the CSs of Figures 4 and 6 tell us that bipolar (and bipolar core) PNs have a larger fraction of massive CSs (i.e., massive progenitors) than round and elliptical PNs, the result is not as sharp as previous results have shown. Our approach is accurate, yet it lacks true luminosity error bars, as the distances to the nebulae were derived in a statistical way. Furthermore, the Zanstra analysis implies the assumption that the CS radiates as a blackbody. It is worth noting that, to date, no CS mass has been derived without strong assumptions on the distances, on the ionized masses, or on model-dependent parameters. Our conclusion is that, to date, there is not a reliable way to measure the mass of Galactic PNs or their stellar progenitors, although our analysis seems to indicate that asymmetric PNs were formed from more massive progenitors, on average.

Third, we have investigated the progenitors' populations of the different morphological PN types: higher mass progenitors should have different chemistry both from the evolutionary dredge-ups and from the different chemistry at star formation. We found that the helium and nitrogen (and very marginally oxygen) average abundances in bipolar and bipolar core PNs are larger than the corresponding values in round and elliptical PNs. In agreement with the theory of stellar evolution, we confirm that B and BC PNs have more massive progenitors than E and R PNs.

On the other hand, the average neon and argon abundances are almost identical in round, elliptical, and bipolar PNs (bipolar core PNs do show high neon and low argon abundances, but the sample size is very small for statistical significance). This result is in contrast to a study of LMC PNs (Stanghellini, et al. 2000), where bipolar (and bipolar core) PNs have higher neon and argon abundances than round and elliptical PNs. We know that there is a larger fraction of bipolar PNs in the LMC than in the Galaxy (Shaw et al. 2001), and that Galactic bipolar PNs tend to be located in the Galactic plane, where the interstellar extinction is maximum. It seems then reasonable that the observed Galactic bipolar PNs are local, so their neon and argon abundances reflect the local environment at formation, rather than the average Galactic disk environment.

Last, we conclude that the study of the statistical correlations between CSs and PN morphology has probably reached, in this form, its exploitation. By increasing the statistical significance and the quality of the analyzed sample we do not get sharper results on the statistical correlations between CSs and PNs. The future of this type of studies is, in our view, two-fold. On one hand, the acquisition and interpretation of CS spectra, and at the same time, the development and improvement of non-LTE stellar models, is essential to acquire the necessary stellar data for a non-biased analysis. On the other hand, better nebular analysis on the individual objects will improve the knowledge of reddening to the 
individual PNs, and their global elemental abundances, possibly extending the analysis to UV spectroscopy, i.e., to reliable carbon abundances. High resolution spectroscopy on the PNs will also be essential to confirm the morphology of some uncertain cases, where the spatial dimension of the velocity is essential.

A continuing study of those extra-Galactic PNs whose morphologies are detectable with today's technology (e.g., the LMC, the SMC, and the nearby dwarf galaxies observed with $H S T$ ) will have the double benefit of minimizing the ill-effects of the unknown Galactic PN distance scale, and those of the poorly known reddening, and of allowing us to extend and integrate our knowledge of the final phases of intermediate-mass stars to Galaxies of different types and metallicities. The emerging picture is that PNs generated by more massive progenitors, those that have been ejected by disk population stars, seem to be bipolar in shape, while smaller mass stars give birth to round PNs. Elliptical PNs are produced by stars in a wider mass range. This result is in agreement with the findings of the PN distribution within the Galaxy, and with the relative $\mathrm{N} / \mathrm{H}$ and $\mathrm{He} / \mathrm{H}$ abundance of the Galactic PNs sample. It bears on the formation of PNs of different morphological types, and it agrees with bipolar PNs to be formed by the evolution of larger mass stars than round and elliptical PNs. While both single and binary star evolutionary models can account for bipolar PN formation, via common envelope evolution, magnetic fields, and fast stellar rotation, the binary scenario alone would not comply with our results.

It is a pleasure to thank Dick Shaw, Bruce Balick, Stacy Palen, Chris Blades, and Max Mutchler for participating in our collective PN morphology classification experiment. Mark Dickinson is thanked for his help in the statistical analysis. 
Table 1. References

\begin{tabular}{ll}
\hline \hline Physical parameter & Reference \\
\hline Stellar magnitude & Abell (1966) \\
& Capellaro et al. (1990) \\
& Dengel, Harlt, \& Weinberger (1980) \\
& Gathier \& Pottash (1988) \\
& Harlt \& Weinberger (1987) \\
& Heap \& Hintzen (1990) \\
& Jacoby \& Kaler (1989) \\
& Kaler (1983) \\
& Kaler \& Feibelman (1985) \\
& Kaler, Shaw, \& Kwitter (1990) \\
& Kohoutek (1979) \\
& Koornneef \& Pottasch (1998) \\
& Kostjakova et al. (1968) \\
& Kwitter, Jacoby, \& Lydon (1998) \\
& Liebert et al. (1988) \\
& Lutz (1977) \\
& Shaw \& Kaler (1985) \\
& Turatto et al. (1990) \\
& Tylenda et al. (1991) \\
& Acker et al. (1992) \\
& Cahn, Kaler, \& Stanghellini (1992) \\
& Pottash \& Zijlstra (1994) \\
& Wendker (1995) \\
& Acker et al. (1992) \\
& Aller (1994) \\
& Cahn, Kaler, \& Stanghellini (1992) \\
& Cuisinier, Acker, \& Köppen (1996) \\
& Aller (1994) \\
& Cuisinier, Acker, \& Köppen (1996) \\
& Cahn, Kaler, \& Stanghellini (1992) \\
& De Marco \& Crowther (1999) \\
Extinction constang & \\
$\operatorname{Fog}\left(F_{H \beta}\right)$ & cm)
\end{tabular}


Table 1-Continued

\begin{tabular}{ll}
\hline \hline Physical parameter & Reference \\
\hline & Stasinska et al. (1992) \\
Tylenda et al. (1992) \\
\hline
\end{tabular}


Table 2. Morphology, temperatures, and luminosities

\begin{tabular}{|c|c|c|c|c|c|}
\hline name & morph. & $\log \mathbf{T}_{\mathrm{eff}}$ & $\Delta_{\log \mathrm{T}}$ & $\log \mathbf{L} / \mathbf{L}_{\odot}$ & $\Delta_{\log \mathrm{L}}$ \\
\hline $\mathrm{A} 4^{\mathrm{a}}$ & $\mathrm{R}$ & 5.114 & 0.302 & 1.717 & 0.308 \\
\hline $\mathrm{A} 8^{\mathrm{a}}$ & $\mathrm{R}$ & 5.187 & 0.301 & 1.932 & 0.302 \\
\hline $\mathrm{A} 12^{\mathrm{a}, \mathrm{b}}$ & $\mathrm{R}$ & 5.214 & 0.301 & 2.497 & 0.302 \\
\hline A16 & $\mathrm{R}$ & 4.936 & 0.040 & 1.800 & 0.142 \\
\hline $\mathrm{A} 20^{\mathrm{b}}$ & $\mathrm{R}$ & 5.012 & 0.033 & 2.744 & 0.105 \\
\hline A 28 & $\mathrm{R}$ & 4.967 & 0.301 & 1.269 & 0.305 \\
\hline A30 & $\mathrm{R}$ & 4.874 & 0.013 & 2.600 & 0.068 \\
\hline A33 & $\mathrm{R}$ & 4.979 & 0.022 & 2.257 & 0.063 \\
\hline A39 & $\mathrm{R}$ & 4.953 & 0.021 & 1.934 & 0.078 \\
\hline $\mathrm{A} 50^{\mathrm{a}}$ & $\mathrm{R}$ & 5.178 & 0.301 & 2.027 & 0.301 \\
\hline $\mathrm{A} 53^{\mathrm{a}, \mathrm{b}}$ & $\mathrm{R}$ & 5.215 & 0.306 & 2.356 & 0.333 \\
\hline A71 & $\mathrm{R}$ & 5.113 & 0.015 & 1.830 & 0.042 \\
\hline $\mathrm{A} 83^{\mathrm{a}}$ & $\mathrm{R}$ & 4.386 & 0.211 & 2.082 & 3.513 \\
\hline BV5-3 & $\mathrm{R}$ & 4.925 & 0.026 & 2.447 & 0.113 \\
\hline Ba1 & $\mathrm{R}$ & 5.058 & 0.008 & 2.126 & 0.025 \\
\hline Cn3-1 & $\mathrm{R}$ & 4.809 & 0.070 & 3.893 & 0.281 \\
\hline H3- $29^{\mathrm{a}}$ & $\mathrm{R}$ & 5.954 & 0.301 & 2.496 & 0.301 \\
\hline $\mathrm{H} 3-75^{\mathrm{a}}$ & $\mathrm{R}$ & 5.099 & 0.301 & 1.987 & 0.301 \\
\hline $\mathrm{He} 1-4^{\mathrm{c}}$ & $\mathrm{R}$ & 5.133 & 0.053 & 3.300 & 0.106 \\
\hline $\mathrm{He} 1-5^{\mathrm{a}}$ & $\mathrm{R}$ & 4.894 & 0.301 & 0.000 & $\ldots$ \\
\hline $\mathrm{He} 2-432^{\mathrm{a}}$ & $\mathrm{R}$ & 4.305 & 0.190 & 4.713 & 3.159 \\
\hline IC $1454^{\mathrm{c}, \mathrm{d}}$ & $\mathrm{R}$ & 5.067 & 0.301 & 1.760 & 0.304 \\
\hline IC $4593^{\mathrm{d}}$ & $\mathrm{R}$ & 4.450 & 0.014 & 3.472 & 0.041 \\
\hline $\mathrm{K} 1-7^{\mathrm{a}}$ & $\mathrm{R}$ & 5.038 & 0.301 & 2.100 & 0.301 \\
\hline $\mathrm{K} 1-9^{\mathrm{a}}$ & $\mathrm{R}$ & 5.194 & 0.301 & 1.295 & 0.302 \\
\hline K1-14 & $\mathrm{R}$ & 4.823 & 0.021 & 3.351 & 0.117 \\
\hline $\mathrm{K} 1-20^{\mathrm{a}}$ & $\mathrm{R}$ & 5.011 & 0.301 & 1.349 & 0.301 \\
\hline $\mathrm{K} 3-2^{\mathrm{a}}$ & $\mathrm{R}$ & 4.267 & 0.178 & 4.790 & 2.953 \\
\hline $\mathrm{K} 3-27^{\mathrm{a}}$ & $\mathrm{R}$ & 5.494 & 0.301 & 2.499 & 0.303 \\
\hline $\mathrm{K} 3-51^{\mathrm{a}}$ & $\mathrm{R}$ & 5.534 & 0.301 & 2.946 & 0.303 \\
\hline $\mathrm{K} 3-56^{\mathrm{a}}$ & $\mathrm{R}$ & 5.449 & 0.301 & 0.000 & $\ldots$ \\
\hline $\mathrm{K} 3-7^{\mathrm{a}}$ & $\mathrm{R}$ & 4.179 & 0.145 & 1.931 & 2.365 \\
\hline K3-73 & $\mathrm{R}$ & 5.049 & 0.008 & 1.779 & 0.022 \\
\hline K3-81 & $\mathrm{R}$ & 4.814 & 0.012 & 3.641 & 0.069 \\
\hline $\mathrm{KjPn} 1^{\mathrm{a}}$ & $\mathrm{R}$ & 5.156 & 0.301 & 2.055 & 0.301 \\
\hline $\mathrm{LSA}^{\mathrm{a}}$ & $\mathrm{R}$ & 4.316 & 0.193 & 2.926 & 3.212 \\
\hline M1- $77^{\mathrm{d}}$ & $\mathrm{R}$ & 4.320 & 0.006 & 4.430 & 0.030 \\
\hline $\mathrm{M} 1-80^{\mathrm{a}}$ & $\mathrm{R}$ & 5.226 & 0.301 & 2.748 & 0.302 \\
\hline$M 4-18^{d}$ & $\mathrm{R}$ & 4.432 & 0.010 & 3.576 & 0.034 \\
\hline NGC 2242 & $\mathrm{R}$ & 5.025 & 0.033 & 2.778 & 0.100 \\
\hline NGC 2438 & $\mathrm{R}$ & 5.175 & 0.082 & 2.055 & 0.134 \\
\hline NGC 3587 & $\mathrm{R}$ & 5.049 & 0.018 & 1.606 & 0.053 \\
\hline NGC 6852 & $\mathrm{R}$ & 5.130 & 0.014 & 2.446 & 0.035 \\
\hline NGC 6879 & $\mathrm{R}$ & 4.835 & 0.026 & 3.617 & 0.130 \\
\hline NGC 6884 & $\mathrm{R}$ & 4.902 & 0.057 & 3.273 & 0.226 \\
\hline NGC $6894^{\mathrm{a}}$ & $\mathrm{R}$ & 4.991 & 0.302 & 2.444 & 0.306 \\
\hline NGC 7094 & $\mathrm{R}$ & 4.874 & 0.009 & 3.058 & 0.042 \\
\hline NGG6842 & $\mathrm{R}$ & 4.994 & 0.031 & 2.782 & 0.105 \\
\hline
\end{tabular}


Table 2-Continued

\begin{tabular}{|c|c|c|c|c|c|}
\hline name & morph. & $\log \mathbf{T}_{\mathrm{eff}}$ & $\Delta_{\log \mathrm{T}}$ & $\log \mathrm{L} / \mathrm{L}_{\odot}$ & $\Delta_{\log \mathrm{L}}$ \\
\hline $\mathrm{Na} 1$ & $\mathrm{R}$ & 4.899 & 0.025 & 3.597 & 0.118 \\
\hline $\mathrm{Sa} 4-1^{\mathrm{d}}$ & $\mathrm{R}$ & 4.315 & 0.009 & 3.113 & 0.044 \\
\hline $\operatorname{Sd} 1^{\mathrm{a}}$ & $\mathrm{R}$ & 4.301 & 0.189 & 2.084 & 3.137 \\
\hline St $3-1^{a}$ & $\mathrm{R}$ & 5.134 & 0.301 & 2.144 & 0.301 \\
\hline Vy2-3 & $\mathrm{R}$ & 4.764 & 0.013 & 4.153 & 0.047 \\
\hline We1-5 & $\mathrm{R}$ & 4.945 & 0.011 & 2.904 & 0.042 \\
\hline A2 & $\mathrm{E}$ & 5.124 & 0.030 & 2.431 & 0.081 \\
\hline $\mathrm{A} 3^{\mathrm{a}}$ & $\mathrm{E}$ & 5.377 & 0.304 & 2.306 & 0.322 \\
\hline A13 & $\mathrm{E}$ & 5.174 & 0.110 & 2.759 & 0.181 \\
\hline A18 & $\mathrm{E}$ & 5.050 & 0.019 & 1.818 & 0.054 \\
\hline $\mathrm{A} 19^{\mathrm{a}}$ & $\mathrm{E}$ & 4.310 & 0.191 & 1.330 & 3.181 \\
\hline $\mathrm{A} 21^{\mathrm{d}}$ & $\mathrm{E}$ & 5.062 & 0.079 & 0.932 & 0.045 \\
\hline $\mathrm{A} 43$ & $\mathrm{E}$ & 4.833 & 0.006 & 2.778 & 0.032 \\
\hline A 46 & $\mathrm{E}$ & 4.859 & 0.079 & 2.169 & 0.268 \\
\hline $\mathrm{A} 49^{\mathrm{a}}$ & $\mathrm{E}$ & 4.303 & 0.190 & 2.393 & 3.148 \\
\hline $\mathrm{A} 54^{\mathrm{a}}$ & $\mathrm{E}$ & 4.305 & 0.190 & 2.288 & 3.159 \\
\hline $\mathrm{A} 57^{\mathrm{d}}$ & $\mathrm{E}$ & 4.667 & 0.022 & 1.666 & 0.022 \\
\hline $\mathrm{A} 63^{\mathrm{d}}$ & $\mathrm{E}$ & 4.623 & 0.251 & 1.922 & 4.084 \\
\hline $\mathrm{A} 70^{\mathrm{a}}$ & $\mathrm{E}$ & 5.242 & 0.302 & 1.160 & 0.306 \\
\hline $\mathrm{A} 72$ & $\mathrm{E}$ & 4.972 & 0.013 & 2.517 & 0.044 \\
\hline $\mathrm{A} 75^{\mathrm{a}}$ & $\mathrm{E}$ & 5.463 & 0.301 & 2.580 & 0.304 \\
\hline A 77 & $\mathrm{E}$ & 4.815 & 0.064 & 3.116 & 0.262 \\
\hline A78 & $\mathrm{E}$ & 4.837 & 0.015 & 3.205 & 0.051 \\
\hline A84 & $\mathrm{E}$ & 5.029 & 0.061 & 1.692 & 0.146 \\
\hline Anom $20 \mathrm{H}^{\mathrm{a}}$ & $\mathrm{E}$ & 4.360 & 0.205 & 1.903 & 3.410 \\
\hline $\mathrm{BD}+3036^{b, c}$ & $\mathrm{E}$ & 4.705 & 0.113 & 3.133 & 0.090 \\
\hline CRL618 & $\mathrm{E}$ & 4.394 & 0.213 & 3.921 & 3.546 \\
\hline $\operatorname{Dd} 1^{\mathrm{a}}$ & $\mathrm{E}$ & 5.189 & 0.301 & 1.295 & 0.302 \\
\hline DeHt2 ${ }^{\mathrm{d}}$ & $\mathrm{E}$ & 4.369 & 0.011 & 1.831 & 0.044 \\
\hline $\mathrm{EGB} 4^{\mathrm{d}}$ & $\mathrm{E}$ & 4.616 & 0.023 & 2.278 & 0.031 \\
\hline $\mathrm{Ha} \operatorname{Tr} 14^{\mathrm{a}}$ & $\mathrm{E}$ & 4.225 & 0.164 & 2.473 & 2.695 \\
\hline $\mathrm{He} 1-2^{\mathrm{d}}$ & $\mathrm{E}$ & 4.475 & 0.038 & 3.867 & 0.097 \\
\hline $\mathrm{He} 2-429^{\mathrm{a}}$ & $\mathrm{E}$ & 4.362 & 0.205 & 3.771 & 3.418 \\
\hline $\mathrm{He} 2-430^{\mathrm{a}}$ & $\mathrm{E}$ & 4.378 & 0.209 & 4.054 & 3.485 \\
\hline $\mathrm{He} 2-447^{\mathrm{a}}$ & $\mathrm{E}$ & 5.031 & 0.301 & 2.773 & 0.301 \\
\hline Hu1-1 & $\mathrm{E}$ & 5.055 & 0.066 & 2.686 & 0.176 \\
\hline IC 1295 & $\mathrm{E}$ & 4.993 & 0.301 & 1.817 & 0.301 \\
\hline IC 2003 & $\mathrm{E}$ & 4.954 & 0.028 & 3.445 & 0.109 \\
\hline IC $2149^{\mathrm{c}, \mathrm{d}}$ & $\mathrm{E}$ & 4.483 & 0.008 & 3.014 & 0.023 \\
\hline IC 351 & $\mathrm{E}$ & 5.127 & 0.114 & 3.066 & 0.218 \\
\hline $\mathrm{J} 320^{\mathrm{c}}$ & $\mathrm{E}$ & 4.772 & 0.012 & 3.700 & 0.074 \\
\hline Jn1 & $\mathrm{E}$ & 4.980 & 0.301 & 1.844 & 0.301 \\
\hline JnEr1 & $\mathrm{E}$ & 5.010 & 0.010 & 1.191 & 0.034 \\
\hline K1-16 & $\mathrm{E}$ & 5.690 & 0.264 & 1.786 & 0.298 \\
\hline $\mathrm{K} 1-17^{\mathrm{a}}$ & $\mathrm{E}$ & 5.343 & 0.301 & 1.517 & 0.302 \\
\hline $\mathrm{K} 2-1^{\mathrm{a}}$ & $\mathrm{E}$ & 5.954 & 0.301 & 0.663 & 0.301 \\
\hline $\mathrm{K} 2-5^{\mathrm{d}}$ & $\mathrm{E}$ & 4.813 & 0.034 & 1.611 & 0.015 \\
\hline $\mathrm{K} 3-5^{\mathrm{a}}$ & $\mathrm{E}$ & 5.375 & 0.301 & 2.644 & 0.302 \\
\hline
\end{tabular}


Table 2-Continued

\begin{tabular}{|c|c|c|c|c|c|}
\hline name & morph. & $\log \mathbf{T}_{\mathrm{eff}}$ & $\Delta_{\log \mathrm{T}}$ & $\log \mathbf{L} / \mathbf{L}_{\odot}$ & $\Delta_{\log \mathrm{L}}$ \\
\hline $\mathrm{K} 3-21^{\mathrm{d}}$ & $\mathrm{E}$ & 4.392 & 0.049 & 2.760 & 0.159 \\
\hline $\mathrm{K} 3-57^{\mathrm{a}}$ & $\mathrm{E}$ & 5.441 & 0.301 & 3.264 & 0.302 \\
\hline $\mathrm{K} 3-61^{\mathrm{a}}$ & $\mathrm{E}$ & 4.299 & 0.188 & 3.537 & 3.126 \\
\hline $\mathrm{K} 3-64^{\mathrm{a}}$ & $\mathrm{E}$ & 4.301 & 0.189 & 2.871 & 3.137 \\
\hline K3-68 ${ }^{a}$ & $\mathrm{E}$ & 5.476 & 0.301 & 2.436 & 0.302 \\
\hline $\mathrm{K} 3-76^{\mathrm{a}}$ & $\mathrm{E}$ & 4.265 & 0.178 & 4.240 & 2.939 \\
\hline K3-79a & $\mathrm{E}$ & 4.230 & 0.166 & 3.590 & 2.728 \\
\hline $\mathrm{K} 3-80^{\mathrm{a}}$ & $\mathrm{E}$ & 4.272 & 0.180 & 3.510 & 2.980 \\
\hline $\mathrm{K} 3-82^{\mathrm{a}}$ & $\mathrm{E}$ & 4.427 & 0.220 & 3.291 & 3.657 \\
\hline K3-92 & $\mathrm{E}$ & 4.901 & 0.006 & 2.737 & 0.018 \\
\hline$K 4-5^{\mathrm{a}}$ & $\mathrm{E}$ & 5.329 & 0.301 & 1.703 & 0.302 \\
\hline M1-2 & $\mathrm{E}$ & 4.736 & 0.024 & 2.384 & 0.155 \\
\hline M1-6 & $\mathrm{E}$ & 4.780 & 0.014 & 3.273 & 0.092 \\
\hline $\mathrm{M} 1-64^{\mathrm{a}}$ & $\mathrm{E}$ & 4.500 & 0.234 & 3.015 & 3.862 \\
\hline $\mathrm{M} 1-66^{\mathrm{a}}$ & $\mathrm{E}$ & 5.037 & 0.301 & 3.080 & 0.301 \\
\hline M1-73 & $\mathrm{E}$ & 4.744 & 0.010 & 3.988 & 0.077 \\
\hline $\mathrm{M} 1-78^{\mathrm{a}}$ & $\mathrm{E}$ & 4.375 & 0.208 & 2.851 & 3.471 \\
\hline $\mathrm{M} 2-2^{\mathrm{a}}$ & $\mathrm{E}$ & 5.216 & 0.301 & 3.229 & 0.302 \\
\hline $\mathrm{M} 2-40^{\mathrm{a}}$ & $\mathrm{E}$ & 4.418 & 0.218 & 3.971 & 3.630 \\
\hline $\mathrm{M} 2-44^{\mathrm{a}}$ & $\mathrm{E}$ & 5.307 & 0.301 & 3.191 & 0.302 \\
\hline $\mathrm{M} 2-45^{\mathrm{a}, \mathrm{b}}$ & $\mathrm{E}$ & 4.279 & 0.182 & 4.081 & 3.018 \\
\hline $\mathrm{M} 2-50^{\mathrm{a}}$ & $\mathrm{E}$ & 4.975 & 0.301 & 2.713 & 0.301 \\
\hline M3-3 ${ }^{\mathrm{a}}$ & $\mathrm{E}$ & 5.022 & 0.302 & 2.332 & 0.306 \\
\hline $\mathrm{M} 4-9^{\mathrm{a}, \mathrm{b}}$ & $\mathrm{E}$ & 4.367 & 0.207 & 2.891 & 3.441 \\
\hline $\mathrm{M} 4-11^{\mathrm{a}}$ & $\mathrm{E}$ & 5.359 & 0.301 & 2.153 & 0.302 \\
\hline Me1-1 & $\mathrm{E}$ & 4.806 & 0.030 & 3.969 & 0.164 \\
\hline NGC $40^{\mathrm{c}, \mathrm{d}}$ & $\mathrm{E}$ & 4.476 & 0.017 & 3.219 & 0.044 \\
\hline NGC 1501 & $\mathrm{E}$ & 4.923 & 0.020 & 3.386 & 0.083 \\
\hline NGC $1514^{\mathrm{c}}$ & $\mathrm{E}$ & 4.711 & 0.018 & 4.178 & 0.062 \\
\hline NGC $2022^{c}$ & $\mathrm{E}$ & 5.075 & 0.019 & 3.452 & 0.049 \\
\hline NGC $2392^{\mathrm{c}}$ & $\mathrm{E}$ & 4.870 & 0.007 & 3.828 & 0.034 \\
\hline NGC $6210^{\mathrm{c}}$ & $\mathrm{E}$ & 4.814 & 0.014 & 3.477 & 0.086 \\
\hline NGC $6543^{\mathrm{c}, \mathrm{d}}$ & $\mathrm{E}$ & 4.690 & 0.051 & 3.080 & 0.045 \\
\hline NGC 6720 & $\mathrm{E}$ & 5.148 & 0.026 & 2.856 & 0.059 \\
\hline NGC $6741^{\mathrm{b}}$ & $\mathrm{E}$ & 5.356 & 0.224 & 2.870 & 0.153 \\
\hline NGC $6781^{\mathrm{c}}$ & $\mathrm{E}$ & 5.022 & 0.040 & 2.104 & 0.112 \\
\hline NGC $6826^{\mathrm{c}, \mathrm{d}}$ & $\mathrm{E}$ & 4.511 & 0.034 & 3.321 & 0.074 \\
\hline NGC 6853 & $\mathrm{E}$ & 4.777 & 0.016 & 1.348 & 0.102 \\
\hline NGC $6905^{\mathrm{c}}$ & $\mathrm{E}$ & 5.117 & 0.042 & 3.668 & 0.093 \\
\hline NGC 7008 & $\mathrm{E}$ & 5.701 & 0.254 & 3.063 & 0.301 \\
\hline NGC $7048^{c}$ & $\mathrm{E}$ & 5.256 & 0.301 & 2.222 & 0.304 \\
\hline NGC $7354^{\mathrm{c}}$ & $\mathrm{E}$ & 4.989 & 0.301 & 3.613 & 0.301 \\
\hline NGC $7662^{\mathrm{c}}$ & $\mathrm{E}$ & 5.051 & 0.069 & 3.395 & 0.184 \\
\hline $\mathrm{PB}^{\mathrm{a}}$ & $\mathrm{E}$ & 4.988 & 0.301 & 3.273 & 0.301 \\
\hline $\mathrm{PC} 19^{\mathrm{a}}$ & $\mathrm{E}$ & 4.949 & 0.301 & 3.818 & 0.301 \\
\hline $\mathrm{Pe} 1-15^{\mathrm{d}}$ & $\mathrm{E}$ & 4.422 & 0.036 & 3.616 & 0.111 \\
\hline Pe1-16 & $\mathrm{E}$ & 4.886 & 0.029 & 4.133 & 0.136 \\
\hline $\operatorname{Pe} 1-20^{\mathrm{a}}$ & $\mathrm{E}$ & 5.608 & 0.301 & 2.177 & 0.303 \\
\hline
\end{tabular}


Table 2-Continued

\begin{tabular}{|c|c|c|c|c|c|}
\hline name & morph. & $\log \mathbf{T}_{\text {eff }}$ & $\Delta_{\log \mathrm{T}}$ & $\log \mathbf{L} / \mathbf{L}_{\odot}$ & $\Delta_{\log \mathrm{L}}$ \\
\hline Pe1-21 ${ }^{a}$ & $\mathrm{E}$ & 5.954 & 0.301 & 2.610 & 0.301 \\
\hline Pu1 & $\mathrm{E}$ & 5.002 & 0.041 & 1.801 & 0.130 \\
\hline $\mathrm{Pu} 2^{\mathrm{d}}$ & $\mathrm{E}$ & 4.606 & 0.062 & 1.371 & 0.086 \\
\hline Vy1-1 & $\mathrm{E}$ & 4.730 & 0.006 & 3.687 & 0.041 \\
\hline Vy1-2 & $\mathrm{E}$ & 5.075 & 0.084 & 3.275 & 0.285 \\
\hline WeSb3 ${ }^{\mathrm{d}}$ & $\mathrm{E}$ & 4.599 & 0.248 & 1.344 & 4.050 \\
\hline $\mathrm{A} 79^{\mathrm{a}, \mathrm{c}}$ & $\mathrm{B}$ & 4.389 & 0.212 & 2.404 & 3.527 \\
\hline HDW5 $5^{\mathrm{d}}$ & B & 4.657 & 0.029 & 1.258 & 0.031 \\
\hline $\mathrm{He} 2-428^{\mathrm{a}}$ & B & 4.248 & 0.172 & 2.837 & 2.839 \\
\hline $\mathrm{He} 2-437^{\mathrm{a}}$ & B & 4.378 & 0.209 & 3.313 & 3.485 \\
\hline $\mathrm{K} 3-1^{\mathrm{a}}$ & B & 5.431 & 0.301 & 2.665 & 0.302 \\
\hline $\mathrm{K} 3-4^{\mathrm{a}}$ & B & 4.326 & 0.196 & 2.896 & 3.262 \\
\hline $\mathrm{K} 3-24^{\mathrm{a}}$ & $\mathrm{B}$ & 4.207 & 0.157 & 4.221 & 2.569 \\
\hline $\mathrm{K} 3-34^{\mathrm{a}}$ & $\mathrm{B}$ & 5.162 & 0.301 & 1.858 & 0.301 \\
\hline $\mathrm{K} 3-45^{\mathrm{a}}$ & B & 4.215 & 0.160 & 3.386 & 2.624 \\
\hline $\mathrm{K} 3-46^{\mathrm{a}}$ & B & 4.344 & 0.201 & 2.211 & 3.344 \\
\hline $\mathrm{K} 3-58^{\mathrm{a}}$ & $\mathrm{B}$ & 4.288 & 0.185 & 3.179 & 3.068 \\
\hline K3-91 & B & 5.234 & 0.301 & 1.790 & 0.302 \\
\hline K3-93 ${ }^{\mathrm{a}}$ & B & 5.113 & 0.301 & 2.242 & 0.301 \\
\hline K3-94 ${ }^{\mathrm{a}}$ & B & 5.283 & 0.301 & 2.388 & 0.302 \\
\hline $\mathrm{K} 4-55^{\mathrm{a}}$ & B & 4.301 & 0.189 & 3.338 & 3.137 \\
\hline M1-8 & B & 5.206 & 0.301 & 2.115 & 0.302 \\
\hline M1-57 & B & 5.116 & 0.147 & 3.762 & 0.271 \\
\hline M1-59a & $\mathrm{B}$ & 5.091 & 0.301 & 0.000 & $\ldots$ \\
\hline $\mathrm{M} 1-75^{\mathrm{a}}$ & B & 5.462 & 0.301 & 2.154 & 0.302 \\
\hline $\mathrm{M} 2-46^{\mathrm{a}}$ & $\mathrm{B}$ & 4.246 & 0.171 & 3.495 & 2.824 \\
\hline $\mathrm{M} 2-48^{\mathrm{a}}$ & B & 4.301 & 0.189 & 3.591 & 3.137 \\
\hline $\mathrm{M} 2-52^{\mathrm{a}}$ & $\mathrm{B}$ & 5.603 & 0.301 & 2.970 & 0.303 \\
\hline M3-28 & $\mathrm{B}$ & 4.260 & 0.176 & 3.881 & 2.912 \\
\hline M3-55 & $\mathrm{B}$ & 4.464 & 0.227 & 3.312 & 3.769 \\
\hline $\mathrm{M} 4-14^{\mathrm{a}}$ & $\mathrm{B}$ & 4.378 & 0.209 & 3.243 & 3.485 \\
\hline $\mathrm{M} 4-17^{\mathrm{a}}$ & $\mathrm{B}$ & 5.103 & 0.301 & 2.125 & 0.301 \\
\hline NGC 650 & $\mathrm{~B}$ & 5.123 & 0.043 & 2.162 & 0.091 \\
\hline NGC $2346^{\mathrm{c}}$ & $\mathrm{B}$ & 4.760 & 0.011 & 3.835 & 0.042 \\
\hline NGC $6778^{\mathrm{b}}$ & $\mathrm{B}$ & 5.030 & 0.070 & 2.605 & 0.199 \\
\hline NGC 6881 & $\mathrm{~B}$ & 4.993 & 0.301 & 0.000 & $\ldots$ \\
\hline NGC $7026^{\mathrm{c}}$ & $\mathrm{B}$ & 4.965 & 0.029 & 2.913 & 0.108 \\
\hline $\mathrm{NGC} 7027^{\mathrm{c}}$ & $\mathrm{B}$ & 5.275 & 0.301 & 5.989 & 0.301 \\
\hline $\operatorname{Pe} 1-14^{\mathrm{a}}$ & $\mathrm{B}$ & 4.196 & 0.152 & 4.299 & 2.491 \\
\hline Pe1-17a & $\mathrm{B}$ & 5.410 & 0.301 & 2.462 & 0.302 \\
\hline Sh1-89a & $\mathrm{B}$ & 5.221 & 0.301 & 1.793 & 0.302 \\
\hline Sh2-71 ${ }^{\mathrm{a}}$ & $\mathrm{B}$ & 4.890 & 0.011 & 3.353 & 0.041 \\
\hline A 80 & $\mathrm{BC}$ & 5.114 & 0.014 & 1.886 & 0.035 \\
\hline $\mathrm{A} 82^{\mathrm{C}}$ & $\mathrm{BC}$ & 4.863 & 0.031 & 3.071 & 0.135 \\
\hline $\mathrm{He} 1-1^{\mathrm{a}}$ & $\mathrm{BC}$ & 5.404 & 0.301 & 3.203 & 0.302 \\
\hline He1- $6^{\mathrm{a}}$ & $\mathrm{BC}$ & 5.082 & 0.301 & 2.102 & 0.301 \\
\hline Hu1-2 & $\mathrm{BC}$ & 5.058 & 0.066 & 3.100 & 0.174 \\
\hline K3-36 & $\mathrm{BC}$ & 4.890 & 0.025 & 3.229 & 0.120 \\
\hline
\end{tabular}


Table 2-Continued

\begin{tabular}{|c|c|c|c|c|c|}
\hline name & morph. & $\log \mathbf{T}_{\mathrm{eff}}$ & $\Delta_{\log \mathrm{T}}$ & $\log \mathbf{L} / \mathbf{L}_{\odot}$ & $\Delta_{\log \mathrm{L}}$ \\
\hline K3-63 & $\mathrm{BC}$ & 4.847 & 0.301 & 3.839 & 0.302 \\
\hline $\mathrm{K} 3-79^{\mathrm{a}}$ & $\mathrm{BC}$ & 4.230 & 0.166 & 3.590 & 2.728 \\
\hline $\mathrm{K} 3-84^{\mathrm{a}}$ & $\mathrm{BC}$ & 5.000 & 0.301 & 2.606 & 0.301 \\
\hline M1-7 & $\mathrm{BC}$ & 5.045 & 0.302 & 2.353 & 0.307 \\
\hline M1-79 & $\mathrm{BC}$ & 5.025 & 0.302 & 2.483 & 0.306 \\
\hline $\mathrm{M} 2-51^{\mathrm{c}}$ & $\mathrm{BC}$ & 4.776 & 0.028 & 3.635 & 0.153 \\
\hline M2-53 & $\mathrm{BC}$ & 5.035 & 0.007 & 2.521 & 0.023 \\
\hline $\mathrm{M} 2-55$ & $\mathrm{BC}$ & 5.031 & 0.038 & 1.883 & 0.099 \\
\hline NGC 6058 & $\mathrm{BC}$ & 4.834 & 0.005 & 3.435 & 0.030 \\
\hline NGC $6772^{\mathrm{b}}$ & $\mathrm{BC}$ & 5.147 & 0.042 & 2.160 & 0.094 \\
\hline NGC $6804^{\mathrm{b}}$ & $\mathrm{BC}$ & 4.954 & 0.007 & 3.804 & 0.030 \\
\hline NGC 7139 & $\mathrm{BC}$ & 5.020 & 0.301 & 1.921 & 0.301 \\
\hline $\mathrm{PB} 10^{\mathrm{a}}$ & $\mathrm{BC}$ & 5.343 & 0.301 & 3.612 & 0.302 \\
\hline
\end{tabular}

${ }^{a}$ Temperature, luminosity, and errors for this nebula are from cross-over analysis. The temperatures should be considered upper limits.

${ }^{\mathrm{b}}$ Morphology and dimensions measured from the Schwarz, Corradi, \& Melnick (1992) images.

${ }^{\mathrm{c}}$ Morphology and dimensions measured from the Balick (1987) images.

${ }^{\mathrm{d}}$ Temperature, luminosity, and errors for this nebula are from $\mathrm{H} \mathrm{I}$ Zanstra analysis. 
Table 3. Physical diagnostics. I. Galactic distribution, extinction, and Zanstra temperatures

\begin{tabular}{lccccc}
\hline \hline morph. & $\begin{array}{c}<|b|> \\
{[\mathrm{deg} .]}\end{array}$ & $\begin{array}{c}\langle|z|> \\
{[\mathrm{kpc}]}\end{array}$ & $c_{\beta}$ & $\begin{array}{c}\left.<T_{\text {eff }}^{a}\right\rangle \\
10^{3}[\mathrm{~K}]\end{array}$ & $<T_{\text {eff,HeII }} / T_{\text {eff,HI }}>$ \\
& & & & \\
\hline $\mathrm{R}$ & $12.6[12.6]$ & $0.73[0.7]$ & $0.63[0.73]$ & $95[25]$ & $1.5[0.6]$ \\
$\mathrm{E}$ & $7.2[7.1]$ & $0.38[0.48]$ & $0.95[0.77]$ & $87[27]$ & $1.4[0.6]$ \\
$\mathrm{BC}$ & $6.9[6.9]$ & $0.37[0.53]$ & $1.03[0.56]$ & $97[27]$ & $1.5[0.7]$ \\
$\mathrm{B}$ & $2.9[2.5]$ & $0.21[0.24]$ & $1.39[0.63]$ & $94[29]$ & $1.2[0.5]$ \\
$\mathrm{R}+\mathrm{E}$ & $8.9[9.6]$ & $0.59[0.5]$ & $0.84[0.77]$ & $90[26]$ & $1.43[0.6]$ \\
$\mathrm{B}+\mathrm{BC}$ & $4.1[6]$ & $0.21[0.24]$ & $1.26[0.63]$ & $96[27]$ & $1.3[0.6]$ \\
\hline
\end{tabular}

${ }^{\mathrm{a}} \Delta \log T_{\text {eff }}<0.1$, He II Zanstra temperatures only. 
Table 4. Physical diagnostics. II. Abundances

\begin{tabular}{llllll}
\hline \hline morph. & $<\mathrm{He} / \mathrm{H}\rangle^{a}$ & $\langle\mathrm{O} / \mathrm{H}\rangle$ & $<\mathrm{N} / \mathrm{H}\rangle$ & $<\mathrm{Ne} / \mathrm{H}\rangle$ & $<\mathrm{Ar} / \mathrm{H}>$ \\
\hline $\mathrm{R}$ & $1.10(18)$ & $3.61(16)$ & $1.71(14)$ & $0.96(16)$ & $1.29(7)$ \\
$\mathrm{E}$ & $1.20(33)$ & $3.34(32)$ & $1.51(31)$ & $0.87(32)$ & $1.28(27)$ \\
$\mathrm{BC}$ & $1.39(9)$ & $3.22(8)$ & $1.92(6)$ & $1.36(5)$ & $0.60(4)$ \\
$\mathrm{B}$ & $1.49(16)$ & $4.05(13)$ & $5.66(13)$ & $0.94(11)$ & $1.36(9)$ \\
\hline
\end{tabular}

${ }^{\mathrm{a}} \mathrm{He} / \mathrm{H}$ abundances are multiplied by $10 ; \mathrm{O} / \mathrm{H}, \mathrm{N} / \mathrm{H}$, and $\mathrm{Ne} / \mathrm{H}$ abundances are multiplied by $10^{4} ; \mathrm{Ar} / \mathrm{H}$ abundances are multiplied by $10^{6}$. 


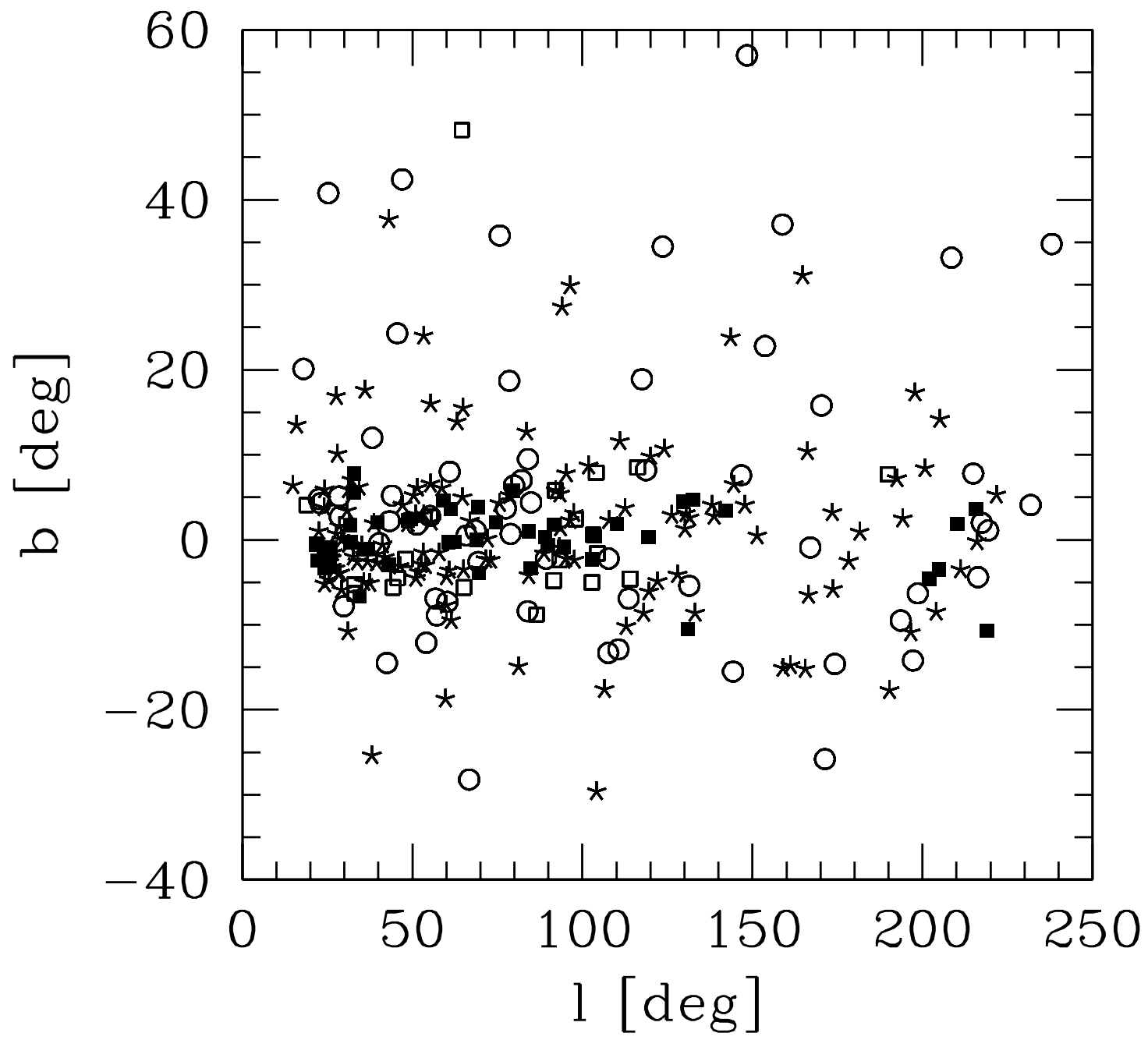

Fig. 1.- Apparent spatial distribution of the sample PNs (Galactic longitude versus Galactic latitude). Open circles: R PNs; asterisks: E PNs; filled squares: B PNs; open squares: BC PNs. 


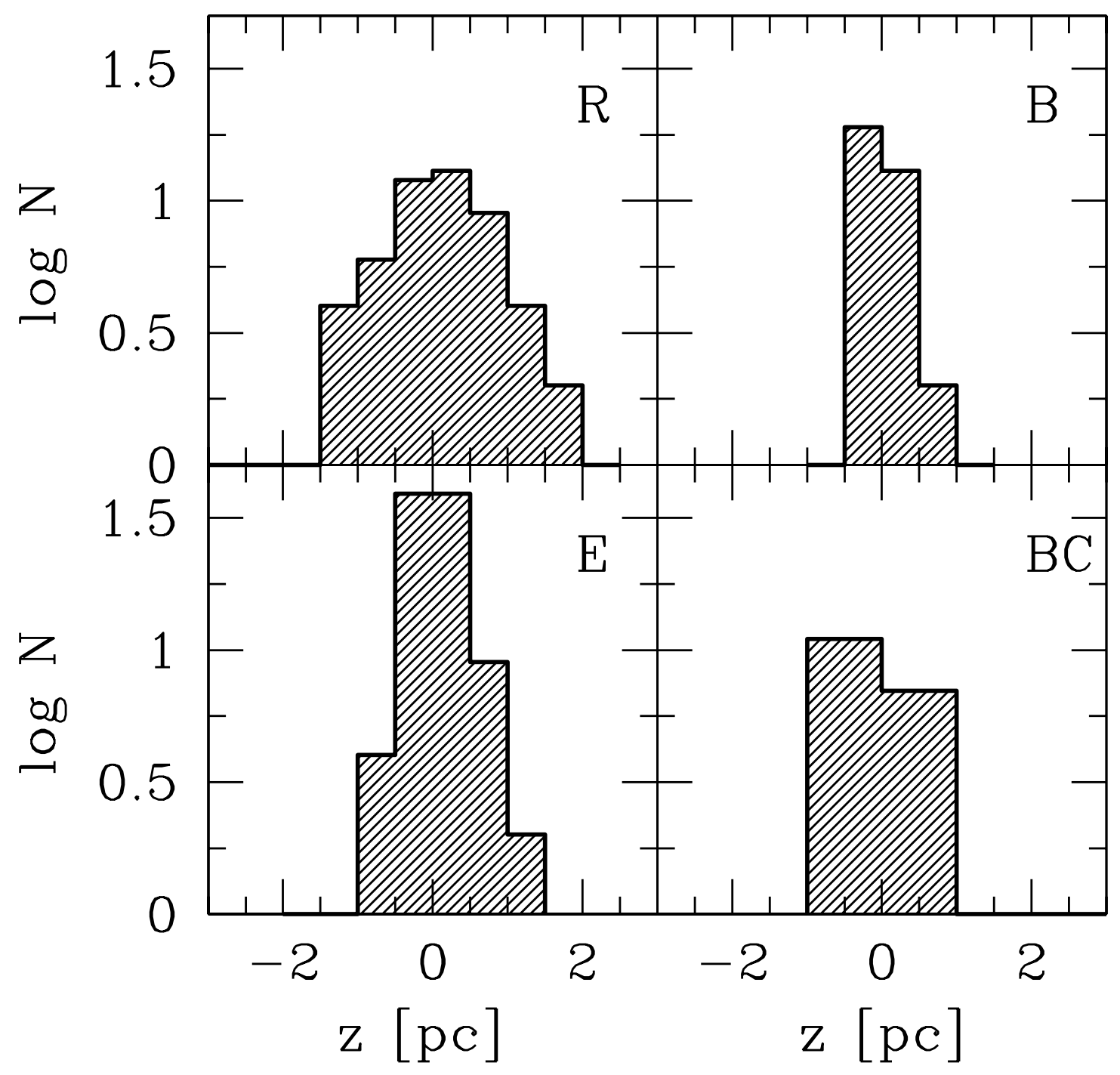

Fig. 2.- Logarithmic distribution of the scale height for the different morphological types. Upper left: round PNs; lower left: upper right: bipolar PNs; lower right: bipolar core PNs. 


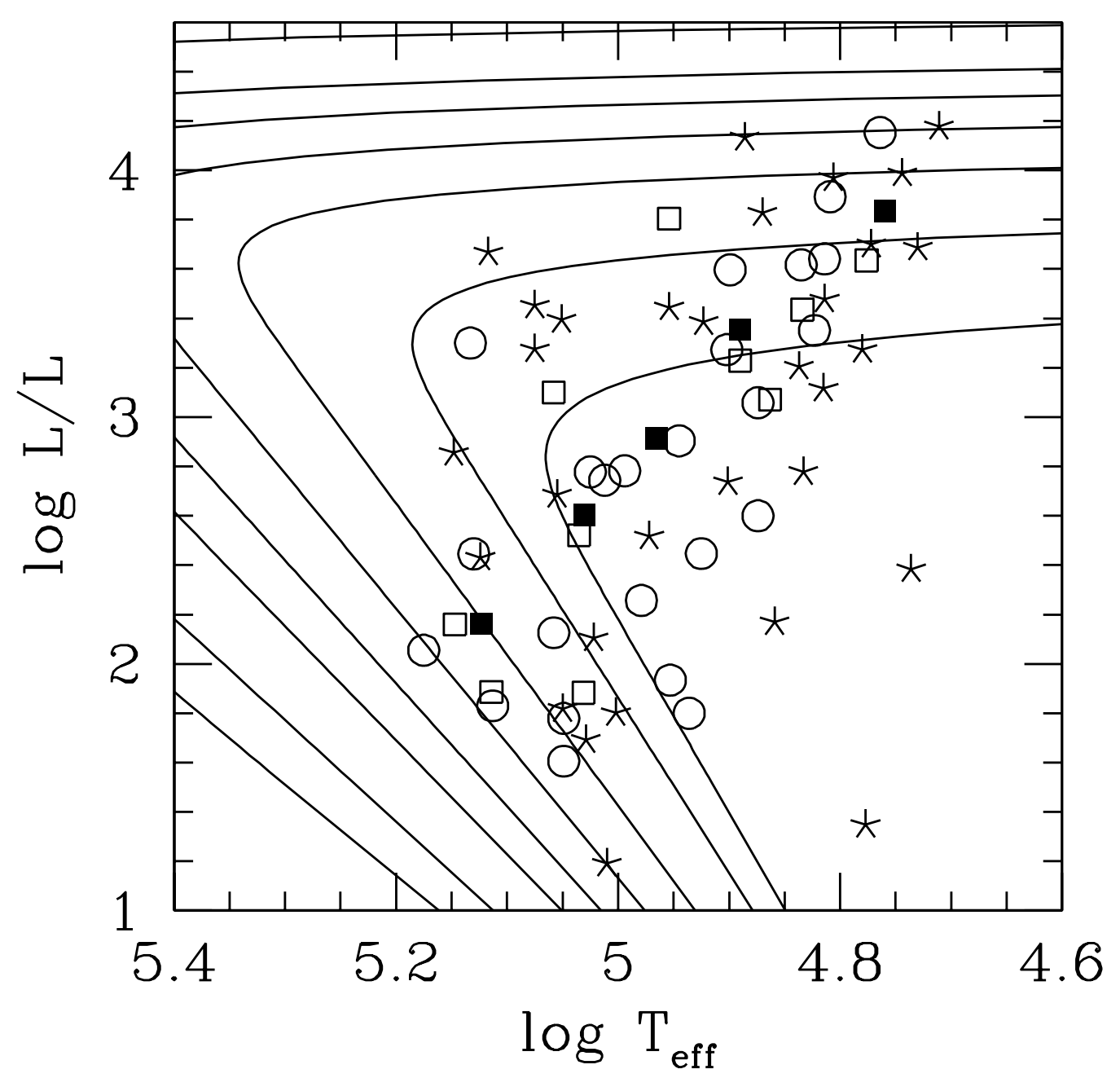

Fig. 3.- Central stars of Planetary Nebulae in the HR diagram. Symbols as in Figure 1. The constraints in the temperature and luminosity errors, in this figure, are $\Delta T_{\text {eff }}<0.1$ dex and $\Delta L<0.3$ dex. We use the He II Zanstra temperatures only. Evolutionary tracks are for $\mathrm{M}=0.55,0.6,0.7,0.8$ 0.9, 1, 1.2, and $1.4 \mathrm{M}_{\odot}$, from Stanghellini \& Renzini (2000). 


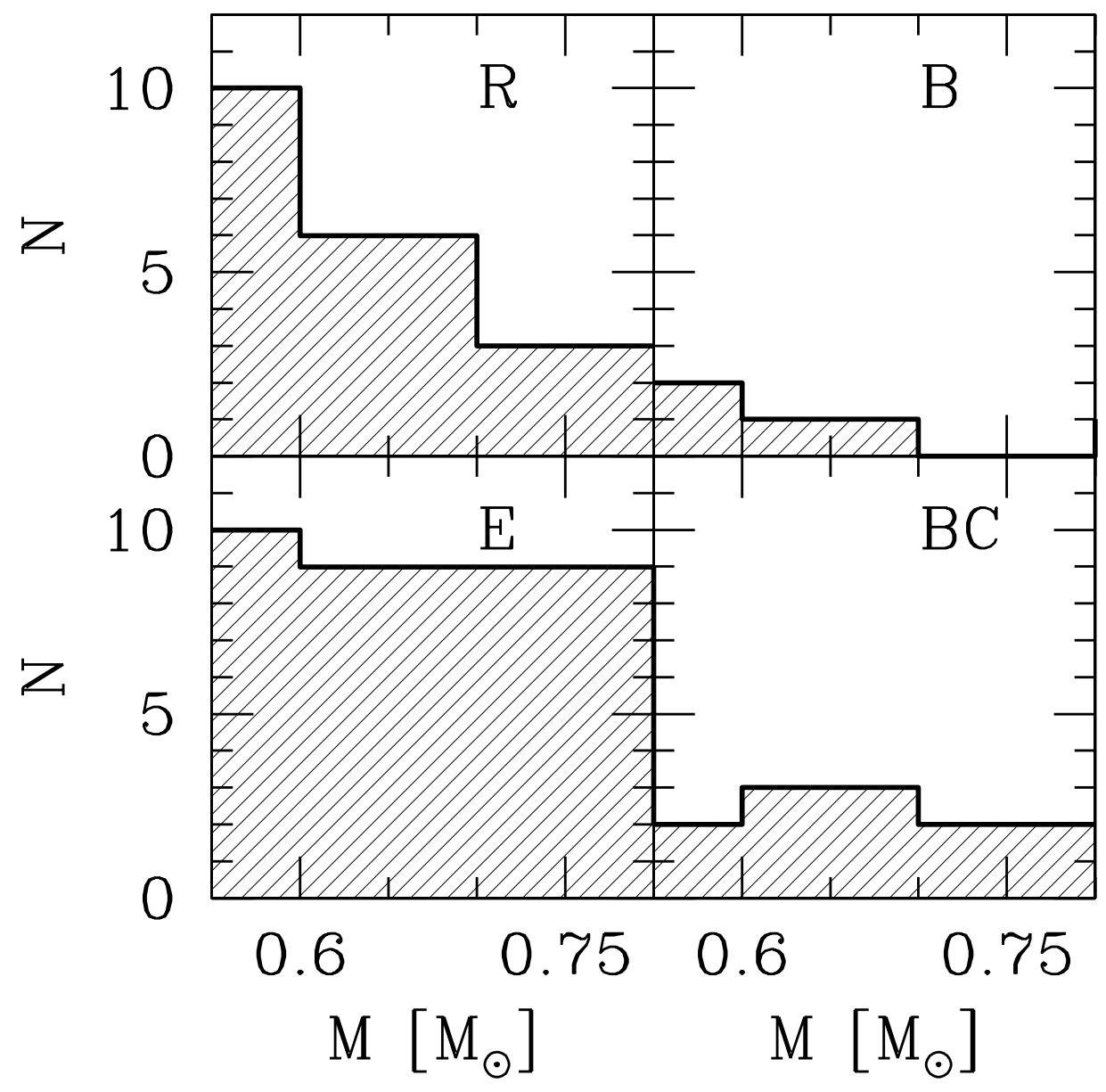

Fig. 4.- Mass distribution of the CSs, from the sample of Figure 3. Upper left: CSs of round PNs; lower left: elliptical PNs; upper right: bipolar PNs; lower right: bipolar core PNs. 


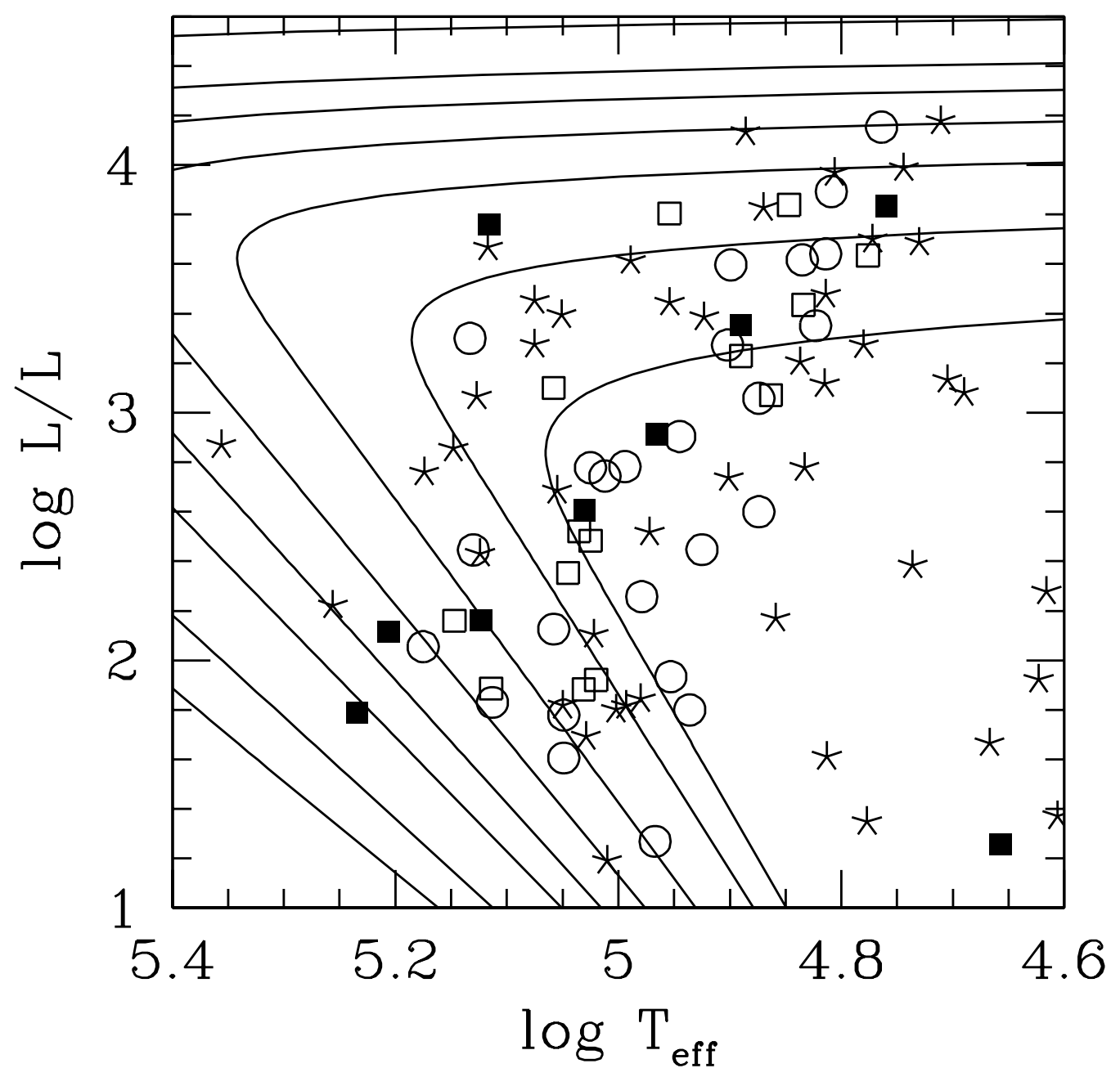

Fig. 5.- Same plot than in Figure 3, except with $\Delta T_{\text {eff }}<0.4$ dex and $\Delta L<0.8$ dex. We use the He II Zanstra temperature and, when those are not available, the $\mathrm{H}$ I Zantsra temperatures. Symbols and tracks as in Figure 3. 


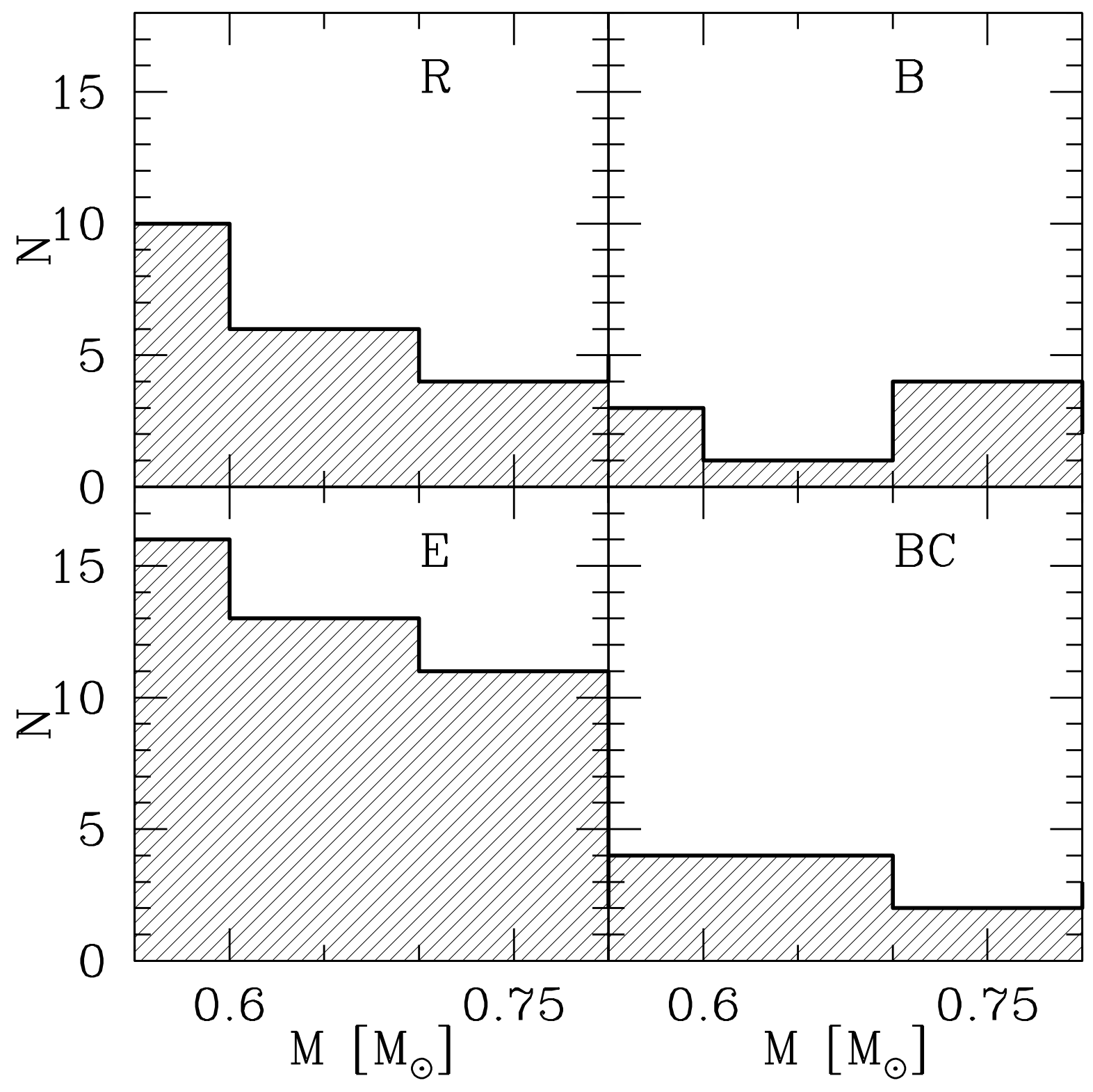

Fig. 6. - Mass distribution of the CSs, from the sample of Figure 5. Upper left: CSs of round PNs; lower left: elliptical PNs; upper right: bipolar PNs; lower right: bipolar core PNs. 


\section{REFERENCES}

Abell, G. O. 1966, ApJ, 144, 259

Acker, A., Ochsenbein, F., Stenholm, B., Tylenda, R., Marcout, J., \& Schohn, C. 1992, Strasbourg-ESO Catalogue of Galactic planetary nebulae (Garching: ESO)

Aller, L. H. 1994, ApJ, 432,427

Amnuel, P. 1995, Ap\&SS, 225, 275

Balick, B. 1987, AJ, 94, 617

Cahn, J. H., Kaler, J. B, \& Stanghellini, L. 1992, A\&AS, 94, 399

Capellaro, E., Turatto, M., Salvadori, L. \& Sabbadin, F. 1990, A\&AS, 86,503

Corradi, R. L. M. \& Schwarz, H. E. 1995, A\&A, 293, 871

Cuisinier, F., Acker, A., \& Köppen, J. 1996, A\&A, 307, 215

De Marco, O. \& Crowther , P. A. 1999, MNRAS, 306, 931

Dengel, J., Harlt, H., \& Weinberger, R. 1980, A\&A, 85, 356

Garca-Segura, G., Langer, N., \& Rozyczka, M. 1999, ApJ, 517, 767

Gathier, R. \& Pottash, S. R. 1988, A\&A, 197,266

Gorny, S. K., Stasinska, G., \& Tylenda, R. 1997, A\&A, 318, 256

Harlt, H. \& Weinberger, R. 1987, A\&AS, 69,519

Heap, S.R. \& Hintzen, P. 1990, ApJ, 353, 200

Iben, I. \& Renzini, A. 1983, ARA\&A, 21, 271

Jacoby, G. H. \& Kaler, J. B. 1989, AJ, 98, 1662

Kaler, J. B. 1983, ApJ, 271, 188

Kaler, J. B. \& Feibelman, W. A. 1985, ApJ, 297, 724

Kaler, J. B., \& Jacoby, G. H. 1989, ApJ, 345, 871

Kaler, J. B., Shaw, R. A., \& Kwitter, K. B. 1990, ApJ, 359, 392 
Kohoutek, L. 1979, IAU Inf. Bull. Var. Stars 1672, 1

Koornneef, J. \& Pottasch, S. R. 1998, A\&A, 335, 277

Kostjakova, E. B., Saveleva, M. V., Dokucheva, O. D., \& Noskova, R. I. 1968, IAU Symposium, 34, Planetary Nebulae, eds. D. Osterbrock \& C. R. O'Dell, 317

Kwitter, K. B., Jacoby, G. H., \& Lydon, T. J., 1998, AJ, 96, 997

Liebert, J., Fleming, T. A., Green, R. F., \& Grauer, A. D. 1988, PASP, 100, 187

Lutz, J. H. 1977, ApJ, 211, 469

Manchado, A., Guerrero, M., Stanghellini, L., \& Serra-Ricart, M. 1996, The IAC Morphological Catalog of Northern Galactic Planetary Nebulae (Tenerife: Instituto de Astrofísica de Canarias)

Manchado, A., Stanghellini, L., \& Guerrero, M. A. 1996, ApJ, 466, L95

Manchado, A., Villaver, E., Stanghellini, L., \& Guerrero, M. A. 2000 , ASP Conf. Ser. 199: Asymmetrical Planetary Nebulae II: From Origins to Microstructures, eds. J. H. Kastner, N. Soker, \& S. Rappaport, 17

Manchado, A., Villaver, E., Stanghellini, L., \& Guerrero, M. A. 2002, in preparation

Peimbert, M. 1978, in IAU Symp. 76, Planetary Nebulae, ed. Y. Terzian (Dordrecht: Reidel), 215

Presse, W. H., Flannery, B. P., Teukolsky, S. A., Vetterling, W. T., 1986, Numerical Recipes, Cambridge University Press

Pottash, S. R. \& Zijlstra, A. 1994, A\&A, 289, 261

Scalo, J. 1998, in ASP Conf. Ser. 142, The Stellar Initial Mass Function, Proc. 38th Herstmonceaux Conf., ed. G. Gilmore \& D. Howell (San Francisciso: ASP), 201

Schwarz, H. E., Corradi, R. L. M., \& Melnick, J. 1992, A\&AS, 96, 23

Schwarz, H. E., Corradi, R. L. M., \& Stanghellini, L. 1993, IAU Symp. 155: Planetary Nebulae, eds. R. Weinberger \& A. Acker ,214

Shaw, R. A. \& Kaler, J. B. 1985, ApJ, 295, 537

Shaw, R. A., Stanghellini, L., Mutchler, M., Balick, B., \& Blades, J. C. 2001, ApJ, 548, 727 
Soker, N. 1997, ApJS, 112, 487

Soker, N. 1998, ApJ, 496, 833

Soker, N. 2002, A\&A, in press

Stanghellini, L., Corradi, R. L. M., \& Schwarz, H. E. 1993, A\&A, 279, 521

Stanghellini, L. 1995, Asymmmetrical Planetary Nebulae, Annales of the Israel Physical Society, Vol. 11, eds. A. Harpaz \& N. Soker, 17

Stanghellini, L., Blades, J. C., Osmer, S. J., Barlow, M. J., \& Liu, X.-W. 1999, ApJ, 510, 687

Stanghellini, L. \& Renzini, A. 2000, ApJ, 542, 308

Stanghellini, L., Shaw, R. A., Balick, B., \& Blades, J. C. 2000, ApJ, 534, L167

Stasinska, G., Tylenda, R. Acker, A., \& Stenholm, B. 1992, A\&A, 266,486

Turatto, M, Capellaro, E., Sabbadin, F., \& Salvadori, L. 1990, AJ, 99, 1170

Tylenda, R., Acker, A., Raytchev, B., Stenholm, B., \& Gleizes, F. 1991, A\&AS, 89 , 77

Tylenda, R., Acker, A., Stenholm, B., \& Köppen, J., 1992, A\&AS, 95,337

Villaver, E., Garcia-Segura, G., \& Manchado, A. 2002, ApJ, in press

Wendker H. J. 1995, A\&AS, 109,177 